\title{
Preliminary Evaluation of Pullulan Nanoparticles Loaded with Valsartan ${ }^{\dagger}$
}

\author{
Ramona-Daniela Pavaloiu 1,2,*, Fawzia Sha'at 1,2, Cristina Hlevca 1, Mousa Sha'at ${ }^{3}$, Claudia Sevcenco ${ }^{1,}$ \\ Maria Petrescu ${ }^{1}$, Mihaela Eremia ${ }^{1}$ and Mișu Moscovici ${ }^{1}$
}

Citation: Pavaloiu, R.-D.; Sha'at, F.; Hlevca, C.; Sha'at, M.; Sevcenco, C.; Petrescu, M.; Eremia, M.; Moscovici, M, Preliminary Evaluation of Pullulan Nanoparticles Loaded with Valsartan. Chem. Proc. 2021, 3, 139. https://doi.org/10.3390/ecsoc-2408428

Academic Editors: Julio A. Seijas and M. Pilar Vázquez-Tato

Published: 14 November 2020

Publisher's Note: MDPI stays neutral with regard to jurisdictional claims in published maps and institutional affiliations.

Copyright: $@ 2020$ by the authors. Licensee MDPI, Basel, Switzerland. This article is an open access article distributed under the terms and conditions of the Creative Commons Attribution (CC BY) license (http://creativecommons.org/licenses/by/4.0/).
1 National Institute for Chemical-Pharmaceutical Research \& Development-ICCF Bucharest, 112, Vitan Avenue, 031299 Bucharest, Romania; fawzya.shaat@gmail.com (F.S.); chlevca@yahoo.com (C.H.); claudiasevcenco@yahoo.com (C.S.); maria.m.petrescu@gmail.com (M.P.); mihaelaceremia@yahoo.com (M.E.); misu_moscovici@hotmail.com (M.M.)

2 Faculty of Applied Chemistry and Materials Science, University Politehnica of Bucharest, 1-7 Gheorghe POLIZU St., 011061 Bucharest, Romania

3 Faculty of Pharmacy, University of Medicine and Pharmacy Grigore T.Popa, 16 Universitatii, 700115 Iasi, Romania; mousa.shaat1@gmail.com

* Correspondence: pavaloiu_daniella@yahoo.com

† Presented at the 24th International Electronic Conference on Synthetic Organic Chemistry, 15 November-15 December 2020; Available online: https://ecsoc-24.sciforum.net/.

\begin{abstract}
The objective of this paper is to develop polymeric nanoparticles loaded with a cardiovascular drug (an angiotensin II receptor blocker, valsartan). Polymeric nanoparticles were prepared via the nanoprecipitation method using pullulan acetate as biodegradable polymeric matrix and Pluronic F127 as a stabilizer. Pullulan acetate was obtained through the chemical modification of pullulan (produced through a fermentation process using the Aureobasidium pullulans strain) with dimethylformamide, pyridine and acetic anhydride. The obtained nanoparticles were characterized in terms of entrapment efficiency, size, and polydispersity index using spectrophotometric and dynamic light scattering techniques. The valsartan-loaded nanoparticles showed a good entrapment efficiency of valsartan, nanometric sizes (lower than $200 \mathrm{~nm}$ ), and a narrow dispersity (polydispersity index below 0.2 ). This research revealed that pullulan and pullulan derivatives show great potential for the production of nanoparticles, with potential application in the delivery of cardiovascular agents.
\end{abstract}

Keywords: pullulan; nanoparticles; valsartan; cardiovascular disease

\section{Introduction}

Cardiovascular diseases represent one of the most major causes of mortality worldwide, accounting for an estimated 17.8 million deaths annually, globally [1]. A need exists for improving the current technologies in cardiovascular therapy and/or developing new strategies for the delivery of cardiovascular drugs.

Over the recent years, polymeric nanoparticles have attracted considerable interest in biomedical applications, (including cardiovascular therapy), due to their numerous advantages, such as: the ability to protect bioactive molecules from premature degradation, the capacity to enhance drugs' bioavailability, absorption and penetration, and to provide the controlled release and targeted delivery of therapeutic agents. Both natural and synthetic polymers have been extensively researched as materials for polymeric nanoparticles production [2,3]. The most commonly used polymers for nanoparticles preparation are polysaccharides, (as natural polymers), and polymethylmethacrylate, poly(cyanoacrylate) (PCA), polycaprolactone (PCL), poly(lactic acid) (PLA), poly-(D,L-glycolic acid) (PGA), poly(lactide-co-glycolide) PLGA (as synthetic polymers [4,5]). Among polysaccharides, pullulan has received a lot of attention from the biomedical community. 
Pullulan is a microbial exopolysaccharide produced by some strains of the polymorphic fungus Aureobasidium pullulans. The structure of pullulan comprises of maltotrose units connected by an $\alpha(1 \rightarrow 4)$ glycosidic bond, whereas consecutive maltotriose units are connected to each other by $\alpha(1 \rightarrow 6)$ glycosidic linkages. Pullulan has nonmutagenic, nontoxic, noncarcinogenic, nonimmunogenic, biocompatible, and biodegradable properties, as well as other functional properties (such as adhesiveness and film formability). Furthermore, pullulan can be easily derivatized in order to widen its applications [6,7]. Hydrophobized pullulan has mostly been used as drug delivery carrier, because pullulan cannot self-associate in aqueous solutions due to its solubility in water. Several pullulan derivatives have been investigated for biomedical applications such as poly(DL-lactideco-glycolide)-grafted pullulan, cholesterol-bearing pullulan, pullulan acetate, perfluoroalkylated pullulan, etc. Pullulan and its derivatives are used in tissue engineering and drug delivery systems due to their excellent physicochemical properties [8,9].

The aim of this research was to investigate the potential of pullulan for the production of nanoparticles with applications in the delivery of cardiovascular agents. To this end, valsartan was used as model cardiovascular drug. Valsartan is an angiotensin II receptor antagonist drug commonly used for the management of hypertension, heart failure, and type 2 diabetes-associated nephropathy, mainly for patients who are unable to tolerate angiotensin-converting enzyme inhibitors. Several clinical trials revealed that valsartan improves cardiovascular diseases outcome, as well as reduces the risk of myocardial infarction, stroke and the progression of heart failure [10,11]. Valsartan has poor solubility in water $\left(1.406 \mathrm{mg} / \mathrm{L}, 25^{\circ} \mathrm{C}\right)$. It is reported that the bioavailability of valsartan is $23-25 \%$ after an oral dose [12]. A way to improve valsartan's poor solubility, reduce its frequent dosing, and increase its bioavailability is by preparing nanoparticles. Therefore, valsartan-loaded, pullulan-based nanoparticles were prepared and characterized in terms of entrapment efficiency, size, and polydispersity index using spectrophotometric and dynamic light scattering techniques.

\section{Materials and Methods}

Pullulan was produced through a fermentation process using the Aureobasidium pullulans strain. Pullulan acetate was obtained via the chemical modification of pullulan with dimethylformamide, pyridine, and acetic anhydride. Valsartan (N-(1-Oxopentyl)-N-[[22(2H-tetrazol-5-yl)[1,12-biphenyl]-4-yl]methyl]-L-valine) and Pluronic F127 (poly(ethylene glycol)-block-poly(propylene glycol)-block-poly(ethylene glycol)) were purchased from Sigma Aldrich (St. Louis, MO, USA). Acetone (analytical grade) was obtained from AdraChim SRL (Bucharest, Romania) and used without further purification. The water used for all experiments was distilled.

\subsection{Preparation of Pullulan Nanoparticles Loaded with Valsartan}

Pullulan nanoparticles were prepared via the nanoprecipitation method. Briefly, pullulan acetate was dissolved in acetone $(5 \mathrm{~mL})$. Valsartan was accurately weighed and solved in a pullulan/acetone solution. Pluronic-F127 $(10 \mathrm{mg})$ was dissolved in distilled water $(15 \mathrm{~mL})$. The organic phase was added dropwise into the aqueous phase solution and stirred magnetically at $1000 \mathrm{rpm}$ at room temperature $\left(25^{\circ} \mathrm{C}\right)$ until the complete evaporation of the organic solvent. The final nanosuspension was centrifuged at 10,000 rpm for $30 \mathrm{~min}$ at $30^{\circ} \mathrm{C}$ to separate the free drug from the formed nanoparticles. The supernatant was carefully siphoned and the precipitate-containing nanoparticles was redispersed in distilled water and filtered through $0.22 \mu \mathrm{m}$ Millex filter membrane. The formulations of the prepared pullulan nanoparticles loaded with valsartan are listed in Table 1. 
Table 1. Formulation of pullulan nanoparticles loaded with valsartan.

\begin{tabular}{cccccc}
\hline $\begin{array}{c}\text { Formulation } \\
\text { Code }\end{array}$ & $\begin{array}{c}\text { Polymer } \\
(\mathbf{m g})\end{array}$ & $\begin{array}{c}\text { Valsartan } \\
(\mathbf{m g})\end{array}$ & $\begin{array}{c}\text { Pluronic F127 } \\
(\mathbf{m g})\end{array}$ & $\begin{array}{c}\text { Drop Rate } \\
(\mathbf{m L} / \mathbf{m i n})\end{array}$ & $\begin{array}{c}\text { Stirring Speed } \\
(\mathbf{r p m})\end{array}$ \\
\hline NP1@VAL & 30 & 5 & 5 & 0.5 & 1000 \\
\hline NP2@VAL & 40 & 5 & 5 & 0.5 & 1000 \\
\hline NP3@VAL & 50 & 5 & 5 & 0.5 & 1000 \\
\hline
\end{tabular}

\subsection{Characterisation of Pullulan Nanoparticles Loaded with Valsartan}

\subsubsection{Entrapment Efficiency}

The entrapment efficiency (EE, \%) was evaluated using an indirect method. The amount of valsartan encapsulated in the pullulan nanoparticles was determined as the difference between the initial amount of valsartan used for nanoparticle preparation and the amount of valsartan present in the supernatant. The percentage of encapsulated drugs was determined spectrophotometrically using a UV-Vis spectrophotometer (JASCO V-630 Spectrophotometer, Jasco International Co., Ltd., Tokyo, Japan) at $250 \mathrm{~nm}$ (the maximum wavelength of valsartan).

\subsubsection{Analysis of Particle Size and Polydispersity Index}

Particle size and polydispersity index were determined using the dynamic light scattering (DLS) method. Particle size and polydispersity index were measured on samples diluted with distilled water $(1: 20)$ at a scattering angle of $90^{\circ}$, a temperature of $25{ }^{\circ} \mathrm{C}$, a solvent refractive index of 1.458 , and a solvent viscosity of $0.8872 \mathrm{cP}$. For each sample the mean value \pm standard deviation of 10 determinations were established. The values reported are the mean value \pm standard deviation for three replicate samples.

\section{Results and Discussion}

The objective of this study was to investigate the potential of pullulan in terms of the production of nanoparticles with applications in the delivery of cardiovascular agents. In order to achieve this aim, polymeric nanoparticles loaded with valsartan were prepared via the nanoprecipitation method using pullulan acetate as a biodegradable polymeric matrix and Pluronic F127 as a stabilizer. The pullulan nanoparticles loaded with valsartan were characterized in terms of entrapment efficiency, size, and polydispersity index.

Particle size is a significant characteristic in determining the performance of a nanoparticulate system because it influences circulating half-life, cellular uptake, and biodistribution. The homogeneity is also a key factor in assessing system performance. The polydispersity index is a measure of the heterogeneity of a sample based on size; a polydispersity index value smaller than 0.1 suggests mono-disperse samples, while values larger than 0.7 are common to a broad size (e.g., polydisperse) distribution of particles. The particle size and polydispersity index of the pullulan nanoparticles loaded with valsartan were determined using the DLS technique. In Table 2, the values of particle size and the polydispersity indices of pullulan nanoparticles are presented. As shown in Table 2, all samples were below $200 \mathrm{~nm}$, and the particle size slightly increased with the increase of pullulan from NP1@VAL (pullulan $30 \mathrm{mg}$ ) $181.50 \pm 0.61 \mathrm{~nm}$ to NP3@VAL (pullulan $30 \mathrm{mg}$ ) $181.50 \pm 0.61 \mathrm{~nm}$. A possible explanation of this fact is that, as the concentration of pullulan increases, the nanosuspension viscosity increases, leading to a decrease in the efficiency of stirring; thus, the diffusion of the organic solvent (acetone) into the aqueous phase is hindered, producing larger droplets, which in turn provide larger particles [13-15]. The polydispersity index values were below 0.2 with little variability between different samples, indicating a good homogeneity of the systems.

The entrapment efficiency of the pullulan nanoparticles loaded with valsartan are presented in Table 3 . All formulations showed a high entrapment efficiency ranging from $(75.13 \pm 0.13) \%$ to $(90.24 \pm 0.14) \%$. The entrapment efficiency is influenced by the 
characteristics of the polymer, drug, surfactant etc. A high entrapment efficiency value is due to the high affinity of the therapeutic agent and the polymer to the same solvent, while a low entrapment efficiency value results from the high affinities of therapeutic agent and polymer to the different solvents [16]. In the present study, an increase in entrapment efficiency with the increase of the pullulan acetate concentration in the formulations can be observed. The increased entrapment efficiency may be due to the higher proportion of polymer present in the formulation.

For the assessment of the stability of the loaded nanoparticles, the samples were kept in amber-colored glass vials and stored at $4{ }^{\circ} \mathrm{C}$ for 3 months (Table 3 ). To assess the stability of samples, the entrapment efficiency parameter was evaluated after different storage periods $(0,1,2$, and 3 months). The pullulan nanoparticles loaded with valsartan were stable for at least 3 months, with approximately the same amount of drug entrapped after 3 months of storage. No aggregation of pullulan nanoparticles loaded with valsartan was observed during storage, probably because the Brownian motion and the diffusion rate for nano-sized formulations are higher than the gravitational-induced sedimentation rate.

Table 2. Particle size and polydispersity index of the pullulan nanoparticles loaded with valsartan.

\begin{tabular}{ccc}
\hline Formulation Code & Size $(\mathbf{n m})$ & Polydispersity Index \\
\hline NP1@VAL & $170.23 \pm 0.52$ & 0.184 \\
\hline NP2@VAL & $173.66 \pm 1.41$ & 0.185 \\
\hline NP3@VAL & $181.50 \pm 0.61$ & 0.187 \\
\hline
\end{tabular}

Table 3. Entrapment efficiency of the pullulan nanoparticles loaded with valsartan.

\begin{tabular}{ccccc}
\hline $\begin{array}{c}\text { Formulation } \\
\text { Code }\end{array}$ & EE, \% & $\begin{array}{c}\text { EE, \% } \\
\text { (1 months) }\end{array}$ & $\begin{array}{c}\text { EE, \% } \\
\text { (2 months) }\end{array}$ & $\begin{array}{c}\text { EE, \% } \\
\text { (3 months) }\end{array}$ \\
\hline NP1@VAL & $75.13 \pm 0.13 \%$ & $74.53 \pm 0.12 \%$ & $74.13 \pm 0.34 \%$ & $74.03 \pm 0.53 \%$ \\
\hline NP2@VAL & $80.25 \pm 0.53 \%$ & $80.05 \pm 1.50 \%$ & $79.85 \pm 0.72 \%$ & $79.25 \pm 1.21 \%$ \\
\hline NP3@VAL & $90.24 \pm 0.14 \%$. & $89.84 \pm 0.24 \%$. & $88.24 \pm 1.02 \%$. & $88.04 \pm 0.14 \%$. \\
\hline
\end{tabular}

\section{Conclusions}

Polymeric nanoparticles were prepared via the nanoprecipitation method using pullulan acetate as biodegradable polymeric matrix, valsartan as the model drug, and Pluronic F127 as a stabilizer. Nanoparticles were characterized in terms of entrapment efficiency, size, and polydispersity index using spectrophotometric and dynamic light scattering techniques. The valsartan-loaded nanoparticles showed a good entrapment efficiency of valsartan, nanometric sizes, and a good homogeneity of their systems. This research revealed that pullulan and pullulan derivatives show great potential for the production of nanoparticles, with application in the delivery of cardiovascular agents.

Author Contributions: Conceptualization, R.-D.P.; M.M.; M.E.; methodology, R.-D.P.; M.E.; software, M.S.; investigation, F.S.; M.E.; M.P.; C.S.; C.H.; writing-original draft preparation, R.-D.P., F.S.; writing - review and editing, R.-D.P. All authors have read and agreed to the published version of the manuscript.

Acknowledgments: This work was financial supported by Romanian National Authority for Scientific Research-ANCSI through Contract PN 1941-04 01.

Conflicts of Interest: The authors declare no conflict of interest.

Institutional Review Board Statement: Not applicable.

Informed Consent Statement: Not applicable. 


\section{References}

1. Virani, S.S.; Alonso, A.; Benjamin, E.J.; Bittencourt, M.S.; Callaway, C.W.; Carson, A.P.; Chamberlain, A.M.; Chang, A.R.; Cheng, S.; Delling, F.N.; et al. Heart disease and stroke statistics-2020 update: A report from the American Heart Association. Circulation 2020, doi:10.1161/CIR.0000000000000757.

2. Mishra, B.; Patel, B.B.; Tiwari, S. Colloidal nanocarriers: A review on formulation technology, types and applications toward targeted drug delivery. Nanomedicine 2010, 6, 9-24.

3. Din, F.U.; Aman, W.; Ullah, I.; Qureshi, O.S.; Mustapha, O.; Shafique, S.; Zeb, A. Effective use of nanocarriers as drug delivery systems for the treatment of selected tumors. Int. J. Nanomed. 2017, 12, 7291-7309.

4. Soppimath, K.; Aminabhavi, T.; Kulkarni, A.; Rudzinski, W. Biodegradable Polymeric Nanoparticles as Drug Delivery Devices. J. Control. Release 2001, 70, 1-20.

5. Rao, J.P.; Geckeler, K.E. Polymer nanoparticles: Preparation techniques and size-control parameters. Prog. Polym. Sci. 2011, 36, 887-913.

6. Seviour, R.; Stasinopoulos, S.; Auer, D.; Gibbs, P.A. Production of Pullulan and other Exopolysaccharides by Filamentous Fungi. Crit. Rev. Biotechnol. 1992, 12, 279-298.

7. Moscovici, M. Present and future medical applications of microbial exopolysaccharides. Front. Microbiol. $2015,6,1012$.

8. Singh, R.; Saini, G.; Kennedy, J. Pullulan: Microbial sources, production and applications. Carbohydr. Polym. 2008, 73, 515-531.

9. Coseri, S.; Spatareanu, A.; Sacarescu, L.; Socoliuc, V.; Stratulat, I.; Harabagiu, V. Pullulan: A versatile coating agent for superparamagnetic iron oxide nanoparticles. J. Appl. Polym. Sci. 2015, 133, 1-9, doi:10.1002/app.42926.

10. Yamashiro, W.; Maeda, K.; Hirouchi, M.; Adachi, Y.; Hu, Z.; Sugiyama, Y. Involvement of transporters in the hepatic uptake and biliary excretion of valsartan, a selective antagonist of the angiotensin II AT1-receptor, in humans. Drug Metab. Dispos. 2006, 34, 1247-1254.

11. Hanna, I.; Alexander, N.; Crouthamel, M.H.; Davis, J.; Natrillo, A.; Tran, P.; Vapurcuyan, A.; Zhu, B. Transport properties of valsartan, sacubitril and its active metabolite (LBQ657) as determinants of disposition. Xenobiotica 2018, 48, 300-313.

12. National Center for Biotechnology Information. PubChem Compound Summary for CID 60846, Valsartan. Available online: https://pubchem.ncbi.nlm.nih.gov/compound/Valsartan (accessed on 10 November 2020).

13. Kheradmandnia, S.; Vasheghani-Farahani, E.; Nosrati, M.; Atyabi, F. The Effect of Process Variables on the Properties of Ketoprofen Loaded Solid Lipid Nanoparticles of Beeswax and Carnauba Wax. Iran. J. Chem. Chem. Eng. 2010, $29,181-187$.

14. Chen, Y.C.; Hsieh, W.Y.; Lee, W.F.; Zeng, D.T. Surface modified PLGA nanoparticles for brain targeting of Bacoside-A. J. Biomater. Appl. 2013, 27, 909-922.

15. Song, X.; Zhao, Y.; Hou, S.; Xu, F.; Zhao, R.; He, J.; Cai, Z.; Li, C.; Chen, Q. Dual agents loaded PLGA nanoparticles: Systematic study of particle size and drug entrapment efficiency. Eur. J. Pharm. Biopharm. 2008, 69, 445-453.

16. Dongming, P.; Huang, K.; Liu, Y.; Lin, S. Preparation of novel polymeric microspheres for controlled release of finasteride. Int. J. Pharm. 2007, 342, 82-86. 